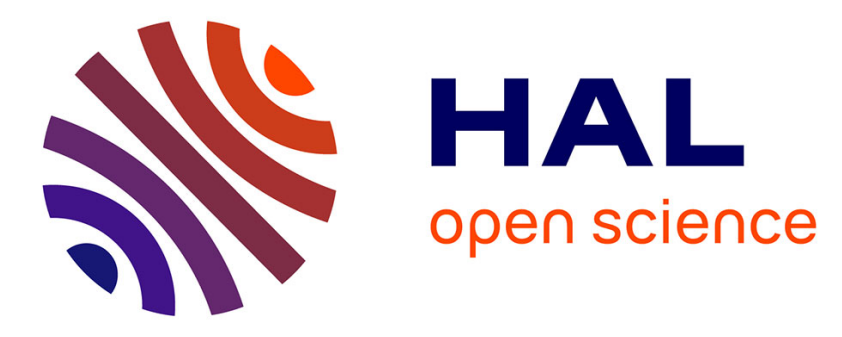

\title{
Forces exerted on a cylinder in near-axial flow
}

Lise Divaret, Pierre Moussou, Julien Berland, Hassan Berro, Olivier Cadot, Olivier Doaré

\section{To cite this version:}

Lise Divaret, Pierre Moussou, Julien Berland, Hassan Berro, Olivier Cadot, et al.. Forces exerted on a cylinder in near-axial flow. Journal of Pressure Vessel Technology, 2014, 136 (5), pp.051306. 10.1115/1.4026567 . hal-01133515

\section{HAL Id: hal-01133515 https://hal.science/hal-01133515}

Submitted on 19 Mar 2015

HAL is a multi-disciplinary open access archive for the deposit and dissemination of scientific research documents, whether they are published or not. The documents may come from teaching and research institutions in France or abroad, or from public or private research centers.
L'archive ouverte pluridisciplinaire HAL, est destinée au dépôt et à la diffusion de documents scientifiques de niveau recherche, publiés ou non, émanant des établissements d'enseignement et de recherche français ou étrangers, des laboratoires publics ou privés. 


\section{Forces exerted on a cylinder in near-axial flow}

\author{
Lise Divaret \\ Laboratoire de Mécanique \\ des Structures Industrielles \\ Durables (LaMSID) \\ UMR EDF CNRS CEA 8193 \\ Clamart, France
}

Email: lise.divaret@edf.fr

Julien Berland

Laboratoire de Mécanique

des Structures Industrielles

Durables (LaMSID)

UMR EDF CNRS CEA 8193

Clamart, France

Email: julien-j.berland@edf.fr

\section{Olivier Cadot}

Unité de Mécanique (UME)

ENSTA ParisTech

Palaiseau, France
Pierre Moussou *

\author{
Laboratoire de Mécanique \\ des Structures Industrielles \\ Durables (LaMSID) \\ UMR EDF CNRS CEA 8193 \\ Clamart, France
}

Email: pierre.moussou@edf.fr
Hassan Berro

Laboratoire de Mécanique

des Structures Industrielles

Durables (LaMSID)

UMR EDF CNRS CEA 8193

Clamart, France

Email: hassan.berro@edf.fr

\section{Olivier Doaré}

Unité de Mécanique (UME)

ENSTA ParisTech

Palaiseau, France

Email: olivier.cadot@ensta-paristech.fr Email: olivier.doare@ensta-paristech.fr 


\begin{abstract}
This study investigates the flow around a cylinder in a near-axial flow at a Reynolds number of 27000. Both CFD calculations and experiments are performed. Time-mean values of lift force coefficient are investigated against the inclination of the cylinder in the domain of low inclinations $\left(<15^{\circ}\right)$. A pressure distribution and flow profiles are also measured and extracted from the CFD calculation results for a characteristic inclination $\alpha=5^{\circ}$. Numerical results for force and pressure show fair agreement with experiments for inclination below $5^{\circ}$ and reveal that at low angles, the lift force is proportional to the angle. In the framework of a quasi-static approach, the instantaneous damping force exerted on a cylinder oscillating in axial flow is equivalent to the normal force exerted on a cylinder placed in an oblique flow
\end{abstract}

\title{
Nomenclature
}

$C_{L} \quad$ Lift coefficient.

$C_{N} \quad$ Normal force coefficient.

$C_{p} \quad$ Pressure coefficient.

D Diameter of the cylinder (m).

$F_{N} \quad$ Normal force per unit length $(\mathrm{N} / \mathrm{m})$.

$F_{D} \quad$ Drag force per unit length $(\mathrm{N} / \mathrm{m})$.

$F_{L} \quad$ Lift force per unit length $(\mathrm{N} / \mathrm{m})$.

L Length of the cylinder (m).

$p_{\infty} \quad$ Far field pressure $(\mathrm{Pa})$.

Re Reynolds number, based on the cylinder diameter and the incoming flow velocity.

$U \quad$ Incoming flow velocity $(\mathrm{m} / \mathrm{s})$.

$U_{\text {parallel }}$ Longitudinal component of the flow velocity $(\mathrm{m} / \mathrm{s})$.

$x \quad$ Distance to the end of the cylinder along the cylinder (m).

$\alpha$ Inclination of the cylinder.

$\rho$ Density of the fluid $\left(\mathrm{kg} / \mathrm{m}^{3}\right)$.

$\theta \quad$ Angular position of the pressure tap.

${ }^{*}$ Address all correspondence to this author. 


\section{INTRODUCTION}

In Pressurized Water Reactors, fuel rods are arranged in light arrays wrapped by grids and subjected to axial flow. A description of the forces exerted upon a cylinder oscillating laterally in an axial flow is needed for seismic design purpose to estimate the flow-induced damping of a fuel assembly. In order to understand the physical phenomena involved, the behavior of only one cylinder oscillating in axial flow is investigated.

The study is focused on the damping force, which is defined as the dissipative force component in the direction of the oscillation and orthogonal to the axis of the cylinder. More precisely, the force acting upon a laterally oscillating cylinder can be expanded in a term proportional to the acceleration, which stands for the added mass effect, and in a term proportional to the velocity, which dissipates energy and hence generates damping in harmonic regime. This dissipative force shall be denoted from now on 'damping force' because it is responsible for fluid damping when a cylinder oscillates in axial flow. In the framework of the quasi-steady approach, i.e., if the lateral velocity of the cylinder is small compared to the axial flow and if the oscillation period is low compared to the inverse of the flow characteristic time $U / D$, the damping force is identical to the normal force exerted upon a cylinder submitted to a near-axial steady flow. One expects this damping force to depend on the axial flow and on the structure velocity.

The normal force for a cylinder oscillating in a fluid at rest has been described by Morison [1,2] as the sum of an added mass force and a drag force. The added mass is a concept [3] which presents the advantage of reducing all the inertial forces to one single coefficient. The Morison expansion can serve as a basis for the description of the forces exerted upon a cylinder oscillating in a axial flow [4]. In the case of a cylinder placed in an oblique flow, Taylor [5] has shown that for oblique flow with angles higher than $20^{\circ}$, the axial component of the fluid velocity has no influence (cross-flow principle). Ersdal \& Faltinsen [6] defined three different cases: for angles lower than $5^{\circ}$, the cross flow principle does not hold, for angles between $5^{\circ}$ and $20^{\circ}$, the cross flow principle needs to be modified by taking into account the state of the boundary layer and beyond $20^{\circ}$ the cross flow principle holds.

The objective of this paper is to compare the results of experiments carried out at small angles, for a cylinder in an oblique flow to RANS CFD simulations of the experiments. As the cross flow principle [5] is not valid for small angles, the variation of the normal force with the axial and lateral velocities needs to be investigated. The validity of the RANS simulations will be discussed for the velocity field, the wall pressure distribution and the total lift force exerted by the fluid on the cylinder. 


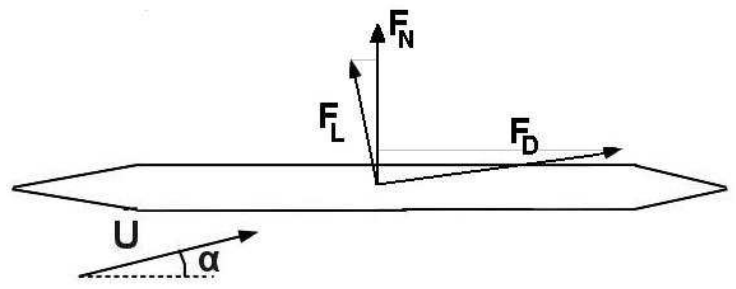

Fig. 1. Cylinder in near axial flow with an angle $\alpha$, definition of the normal force $F_{N}$, the lift force $F_{L}$ and the drag force $F_{D}$.

\section{STATE OF THE ART}

The scientific literature provides very few data about cylinders in near axial flow. Taylor [5] summed up the results of Relf and Powell about the normal force exerted on a cylinder placed in an oblique flow in an air tunnel at angles of inclination between $10^{\circ}$ and $90^{\circ}$. The normal force $F_{N}$ is the force in a direction normal to the cylinder axis and in the plane of the incoming flow velocity and of the cylinder axis (see Fig. 1). In their experiment, the normal force was proportional to the square of the lateral component of the velocity. In other words, the normal force measured for a cylinder placed in an oblique flow of velocity $U$ and angle $\alpha$ is the same as the one exerted upon the same cylinder placed in a cross flow of velocity $U \sin (\alpha)$. This implies that the axial component of the flow velocity has no influence on the normal force coefficient in this range of angles.

$$
F_{N}=\frac{1}{2} C_{N} \rho_{f} D(U \sin \alpha)^{2} \quad \text { for } \alpha>20^{\circ}
$$

This result classically refers to as the cross flow principle. According to equation (1), the normal force coefficient $C_{N}$ is around 1.1, which corresponds to the drag of a cylinder in cross flow.

Ersdal \& Faltinsen [6] recently carried out experiments with a cylinder mounted on a towed carriage. The cylinder was towed at a constant velocity and angle $\alpha$ and the normal force was measured. The experiments were performed by steps of 1 or 2 degrees. Results similar Taylor's one were observed for angles above $20^{\circ}$. For angles between $5^{\circ}$ and $20^{\circ}$, a flow regime transition was observed: the boundary layer which is laminar for high angles becomes turbulent. For practical purposes, this means that the cross flow principle holds, but the normal force coefficient is lower than for a laminar boundary layer. Ersdal $\&$ Faltinsen measured a normal force coefficient $C_{N} \approx 0.8$

For angles lower than $5^{\circ}$, the cross flow principle does not hold. Taylor [5] proposed a model based on the friction force which is now traditionally used at low angles $[7,8]$. Ersdal \& Faltinsen showed that the normal force is proportional to the 


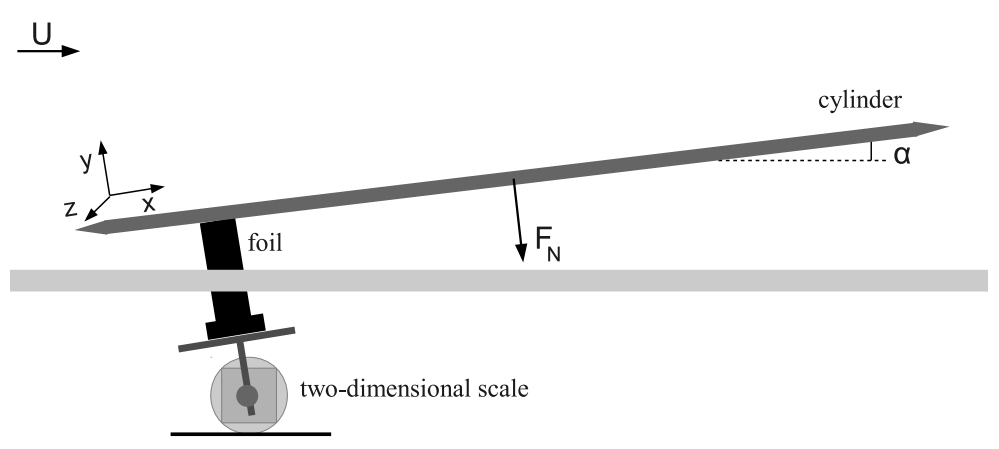

Fig. 2. Experimental setup.

lateral velocity $U \sin (\alpha)(2)$ and observed that the lift force is the dominant term of the normal force.

$$
F_{N}=\frac{1}{2} C_{N} \rho_{f} D U^{2} \sin \alpha \quad \text { for } \alpha<5^{\circ}
$$

In the present work, the description of the fluid forces is focused on the lift force $F_{L}$. For a cylinder in an oblique flow, $F_{L}$ is the fluid force in the direction illustrated in Fig. 1. The non-dimensional lift force coefficient is defined by considering the incoming flow velocity as the reference velocity.

$$
C_{L}=\frac{F_{L}}{\frac{1}{2} \rho_{f} D U^{2}}
$$

\section{EXPERIMENTAL SET UP}

The experiments are all performed in an air tunnel. A cylinder is arranged in a near-axial flow with an angle of inclination $\alpha$, varying from $0.5^{\circ}$ (axis almost parallel to the flow) to $15^{\circ}$. The length of the cylinder is $L=1.2 \mathrm{~m}$, its diameter $D=0.02 \mathrm{~m}$ and the ends of the cylinder are cone-shaped to prevent flow separation. The incoming flow velocity is measured with a Pitot tube, with an accuracy of $\pm 0.1 \mathrm{~m} / \mathrm{s}$.

Three types of experiments are performed in the wind tunnel. The goal of the first experiment shown in Fig. 2 is to measure the forces $F_{L}$ and $F_{D}$ exerted on the cylinder. The cylinder is fixed to a foil mounted on a two-dimensional scale, and a motorized system allows the rotation of the scale. The drag and the lift forces are measured simultaneously and the contribution of the mounting system is subtracted.

In the second experiment, the velocity profiles along the cylinder are measured with the help of a 1D hot wire probe. 


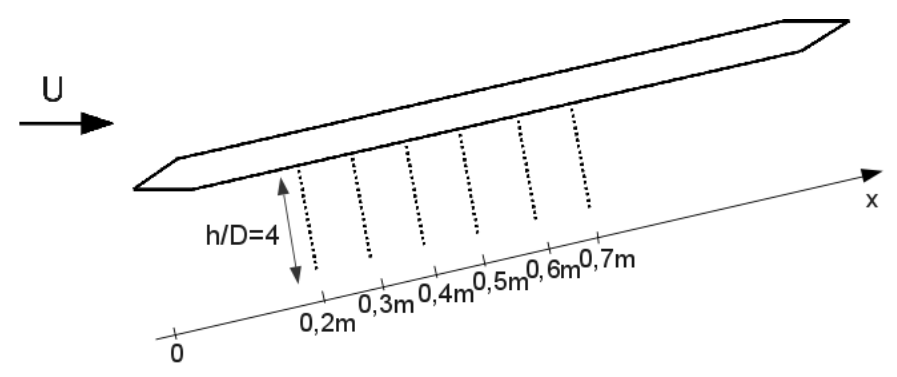

Fig. 3. Position of the velocity profiles in the $y$ direction along the cylinder at an azimuthal angle $\theta=180^{\circ}$.

The profiles are measured in the wake of the cylinder, at an azimuthal angle $\theta=180^{\circ}$. Measurements are performed close to the cylinder wall, by varying the distance in the $y$ direction from 0.4 to $1 \mathrm{~mm}$ by step of $0.1 \mathrm{~mm}$, then from 1 to $5 \mathrm{~mm}$ by step of $1 \mathrm{~mm}$, from 5 to $20 \mathrm{~mm}$ by step of $2.5 \mathrm{~mm}$ and from 20 to $80 \mathrm{~mm}$ by step of $10 \mathrm{~mm}$. The probe is arranged at several locations along the cylinder, as shown in Fig. 3: the distance to the upstream end of the cylinder spans from $0.2 \mathrm{~m}$ to $0.7 \mathrm{~m}$, i.e., $x / L$ varies from 0.17 to 0.58 .

The third experiment consists in measuring the pressure distribution in the cross section in the cylinder. A pressure tap is arranged at a distance $X=0.46 \mathrm{~m}$ from the end of the cylinder $(X / L=0.38)$. By rotating the cylinder around its axis, the pressure distribution around it can be measured. Results are provided for an angle $\alpha$ equal to $5^{\circ}$, and an angle $\theta$ varying from $0^{\circ}$ to $180^{\circ}$ by steps of $15^{\circ}$. The incoming flow velocity is $20 \mathrm{~m} / \mathrm{s}$, which corresponds to a diameter-based Reynolds number equal to 27000 .

The reference frame used in the following is described in Fig. $2:$ the $x$ axis is parallel to the cylinder axis.

\section{CFD PROCEDURE}

In addition to experiments, CFD simulations have been carried out. In order to reduce the calculation time, the fluid domain of the CFD calculations is smaller than the test section of the wind tunnel, but its width is large enough to avoid confinement effects. As for the experiments, the cylinder length is $L=1.2 \mathrm{~m}$ and the cylinder diameter $D=0.02 \mathrm{~m}$.

The fluid fow is calculated using Code Saturne, an EDF in-house open CFD tool [9] based on a collocated finite volume approach. Three dimensional steady RANS simulations are performed with a $k-\omega$ turbulence model. An analysis different turbulence models ranges beyond the scope of this paper, be it enough to mention that a simulations with $k-\varepsilon$ turbulence model provided similar results.

The mesh is built with quadrangle elements only, as shown in Fig. 4. The reference mesh has a number of $1.66 \times 10^{6}$ cells. The cylinder dimensions are the same as in the experiments. The dimensions of the fluid domain are $V=0.92 m \times$ 


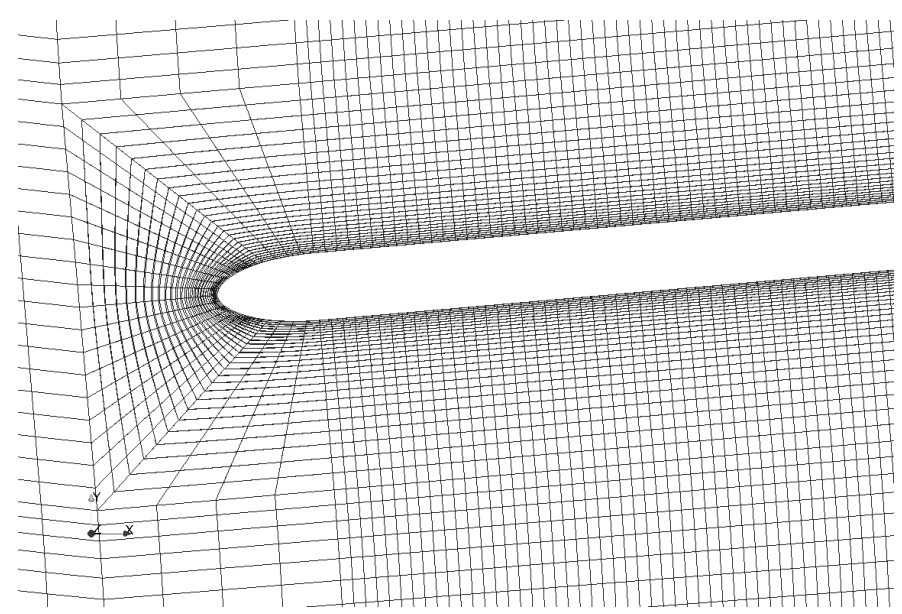

Fig. 4. Mesh, zoom on the end of the cylinder.

$0.32 m \times 1.8 m$. The mesh is refined close to the walls of the cylinder $\left(1<y^{+}<30\right)$ and a two scale wall law is chosen.

The resolution of the velocity field and pressure is directly linked to the size of the mesh. The pressure distribution around the cylinder is hence determined by steps of $8^{\circ}$, which corresponds to about $1 \mathrm{~mm}$. The same resolution is obtained for the velocity profiles close to the cylinder.

\section{LIFT COEFFICIENT}

The lift and drag contributions to the normal force measured with the two-dimensional scale are shown in Fig. 5, with the notations of Fig. 1 : the normal force is essentially due to the lift force. Moreover, the figure shows two different flow regimes : the first shows a linear dependency of the normal force with respect to the yaw angle $\alpha$ for values lower than $5^{\circ}$, and the second exhibits a quadratic dependency for higher values, consistent with the so-called cross-flow principle, and already observed by Ersdal \& Faltinsen [6]. Païdoussis [8] has already pointed out that the normal force coefficient in the linear part could be higher than the one obtained by the projection of the drag force in the normal direction but he attributed this effect to friction. The results presented in Fig. 5 show that the linearity of the normal force is due to the linearity of the lift force in the linear regime.

The experimental results are also compared to the results of the CFD calculations. A qualitative agreement is obtained between the CFD calculations and the measurements, as illustrated by the linear regression on the experimental data plotted in dashed line in Fig. 5, and by the slope estimation in Table 1. Discrepancies appear in the range of angles beyond $5^{\circ}$, but the normal force at small angles is reasonably predicted by the simulations.

The figure 6 shows the variation of the lift component of the normal force $C_{L} \cos \alpha$ with respect to the inclination angle with a log scale. It highlights the transition between the linear and the quadratic behavior around $5^{\circ}$. The reasons for this 


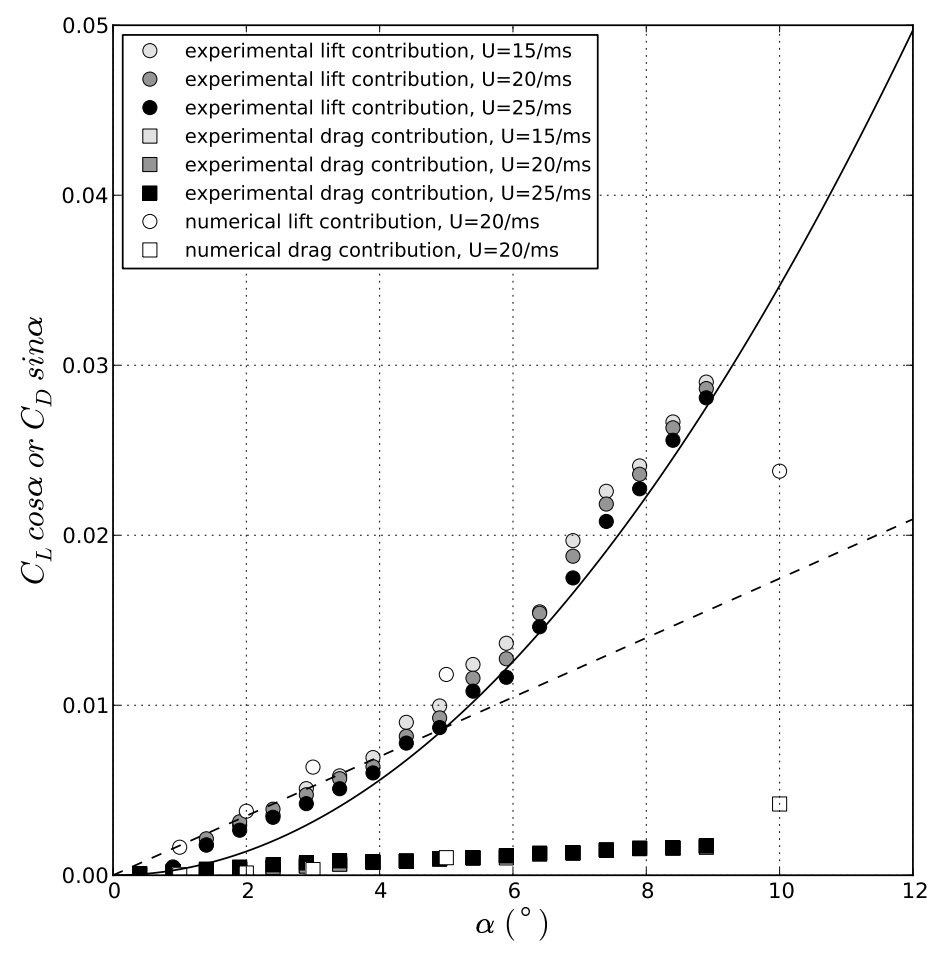

Fig. 5. Lift and drag contributions to the normal force $C_{L} \cos \alpha$ et $C_{D} \sin \alpha$ such as $C_{N}=C_{L} \cos \alpha+C_{D} \sin \alpha$. Comparison between experimental measurements and CFD simulation results. Linear regime for $|\alpha|<5^{\circ}$ and comparison with the cross flow principle for $|\alpha|>5^{\circ}$

Table 1. Slope of the lift coefficient for $\alpha<5^{\circ}$

\begin{tabular}{lll}
\hline & Experiments & Simulations \\
\hline$C_{L} / \alpha[\mathrm{rad}]$ & 0.010 & 0.0125 \\
\hline
\end{tabular}

quadratic behavior are related to flow separation effects that can not be described here, and the discussion is focused on the linear range. The results are reasonably independent on the incoming flow velocity.

\section{FLOW PATTERN}

The observation of the flow pattern with the hot wire reveals no significant r.m.s. velocity further than $1.5 \mathrm{D}$ from the cylinder. The RANS simulations show a similar trend, as presented in Fig. 7. It can hence be assessed that no large unsteady structures are generated by the oblique flow at low angles. More precisely, a gradual deficit of velocity is observed during the 


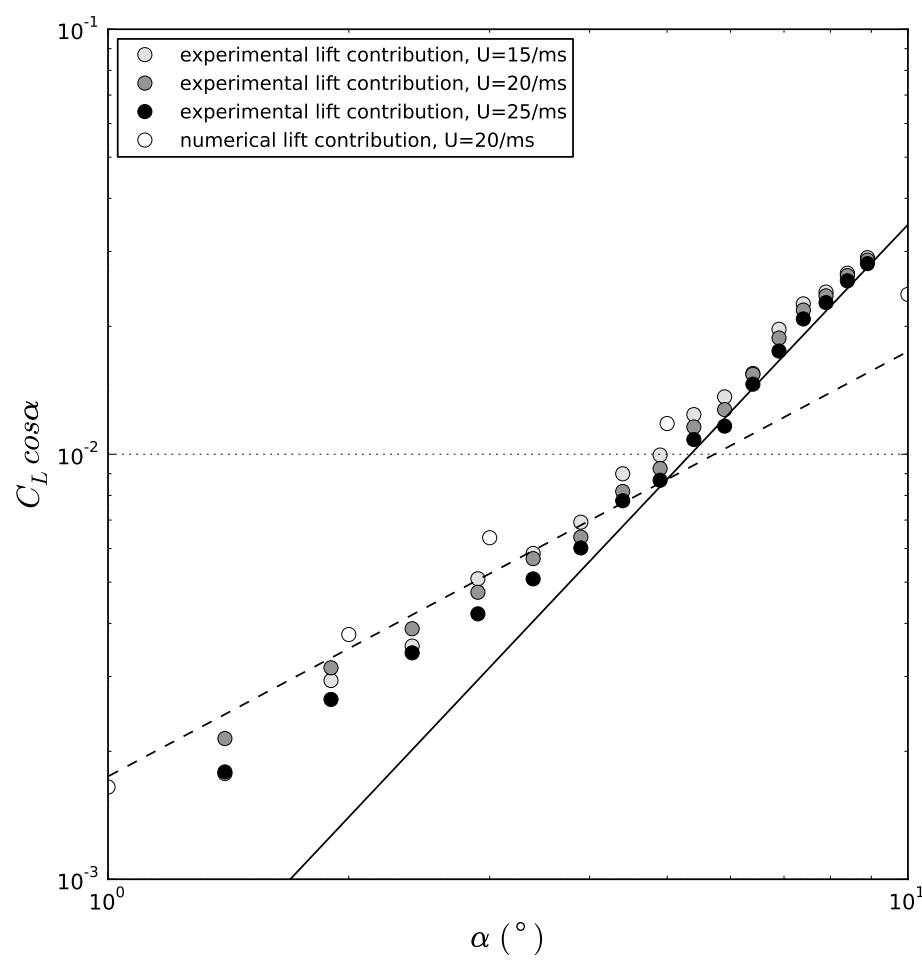

Fig. 6. Experimental and numerical lift contributions to the normal force $C_{L} \cos \alpha$. A transition between the linear and the quadratic regimes is observed.

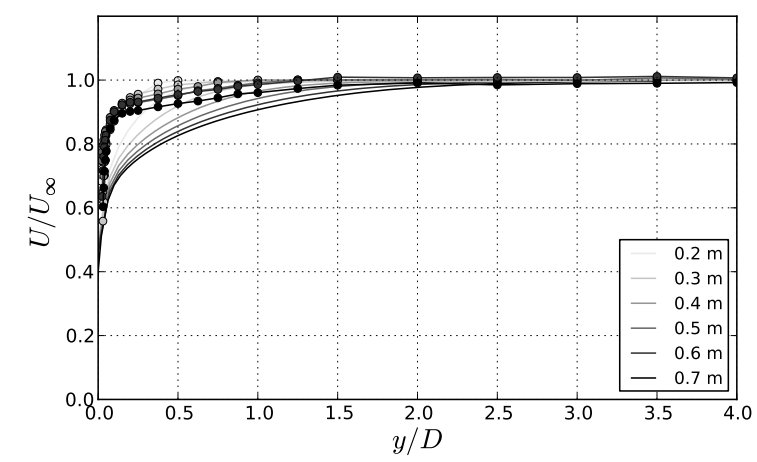

Fig. 7. Velocity profiles at a yaw angle of $5^{\circ}$. $\bullet$ : experiments and $-:$ CFD simulations.

experiments and predicted by the RANS simulation on the lower side $\left(\theta=180^{\circ}\right)$ (see Figs. 8 and 9 ), with a thickness varying from 1.0 to $1.5 D$, whereas the velocity is almost uniform on the upper side $\left(\theta=0^{\circ}\right)$. This highly differs from cross-flow cases $[7,10,11]$, where unsteady vortices are shed along the separation line. This absence of a clear flow separation is a distinct feature of quasi-oblique flow, which does not seem to have been reported up to now.

Some discrepancies are present when comparing the CFD and experimental velocity profiles; the velocity gradient is 


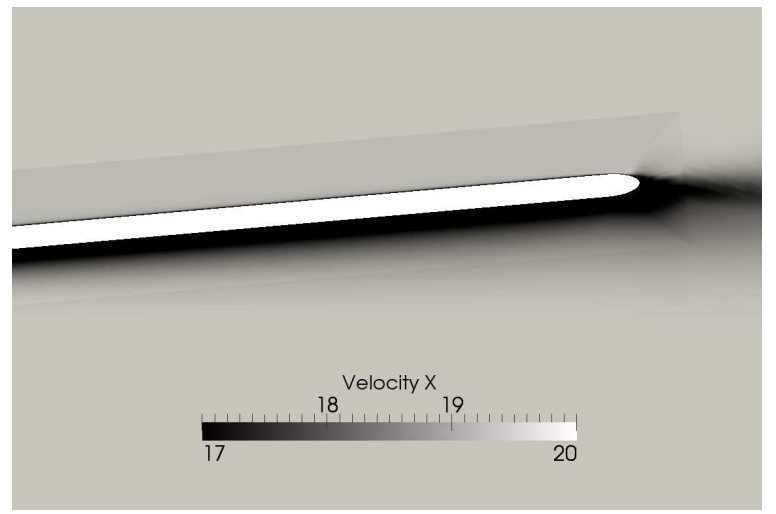

Fig. 8. Velocity in the $x$-direction, cylinder in near-axial flow with a yaw angle $\alpha=5^{\circ}$, view in the plane $(x, y)$.

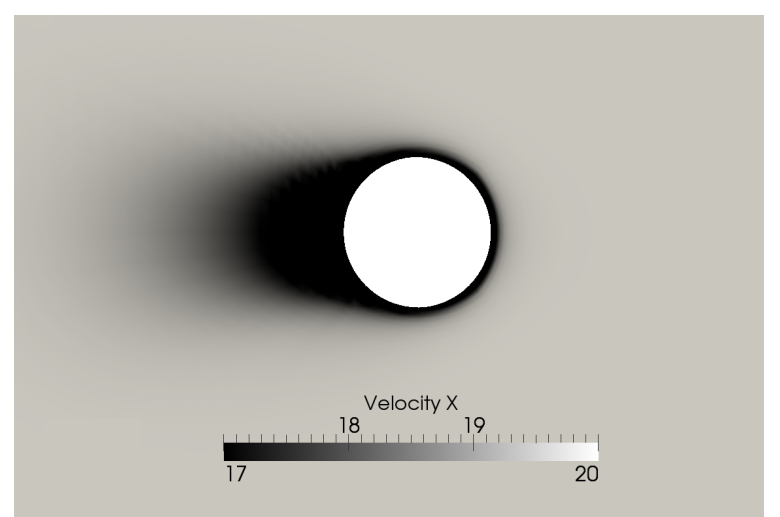

Fig. 9. Velocity in the $x$-direction (defined in Fig.3), cylinder in near-axial flow with a yaw angle $\alpha=5^{\circ}$, view in the plane $(y, z)$ at a distance $x=0.46 \mathrm{~m}$ from the first end of the cylinder.

smaller in the computations, and the deficit area is larger with a thickness varying from 1.5 to $2.5 D$. This seems to indicate that the RANS approach overestimates diffusion effects close to the cylinder wall. This point shall be discussed at longer extent in the discussion section.

Another feature of the oblique flow is the variation of the thickness of the velocity deficit area along the cylinder, which indicates that the flow pattern is not purely two-dimensional.

\section{PRESSURE DISTRIBUTION ALONG THE CYLINDER WALL.}

The coefficient $C_{p}$ in Eq. (4) is defined as a dimensionless pressure difference, with the far field pressure chosen as reference. The Fig. 10 shows the convention for the angular position of the pressure tap on the instrumented cylinder. 


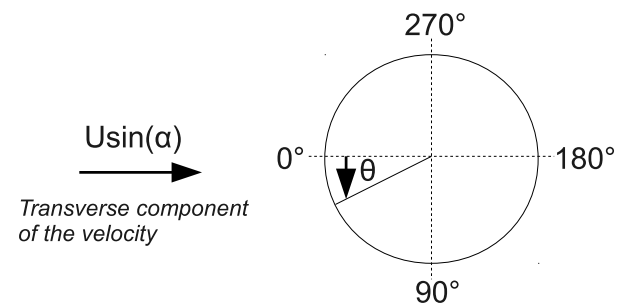

Fig. 10. Angular position of the pressure tap. View in the place $(y, z)$.

$$
C_{p}=\frac{p-p_{\infty}}{\frac{1}{2} \rho_{f} U^{2}}
$$

The experimental and computed pressure distributions are compared in Fig. 11. At $\theta=0^{\circ}$, the pressure coefficient $C_{p}$ is maximal and strictly lower than 1 , which differs from cross flow pressure distributions where a stagnation point generates a value equal to 1 . More precisely, if the velocity were equal to zero at $\theta=0^{\circ}$, a direct application of the Bernoulli equation would lead to $C_{p}=1$. The other way around, as the pressure coefficient is not equal to 1 , one expects a non-vanishing flow velocity parallel to the axis at $\theta=0^{\circ}$ to exist and which can be estimated by applying the Bernoulli equation (5).

$$
U^{2}+\frac{p_{\infty}}{\rho_{f}}=U_{\text {parallel }}^{2}+\frac{p_{\theta=0}}{\rho_{f}}
$$

A value of $U_{\text {parallel }}=19.88 \mathrm{~m} \cdot \mathrm{s}^{-1}$ is obtained, very close to the incoming flow velocity equal to $20 \mathrm{~m} / \mathrm{s}$ and up to the measurement uncertainty. Further work is needed to accurately describe the flow pattern: whether the flow is deviated around the cylinder or convected along the line $\theta=0$ is not clear, and cannot be easily assessed in the framework of the present study.

Between $\theta=0^{\circ}$ and $\theta=80^{\circ}$, the pressure coefficient gradually decreases, as it is the case in cross flow where the velocity increases. It reaches its minimal value at $\theta_{\min }=80^{\circ}$, and increases between $\theta=80^{\circ}$ and $\theta=180^{\circ}$, where it is equal to about -0.005 , a negative value which is consistent with the velocity deficit observed in the previous section.

In Fig. 11, a fairly reasonable agreement is obtained between the experimental and the calculated pressure distribution around the cylinder. The values of the pressure at the upper side $\theta=0^{\circ}$, the lower side $\theta=180^{\circ}$ and at $\theta_{\min }$ are close. However, the minimum angle $\theta_{\min }$ is equal to $80^{\circ}$ in the experiments and to $80^{\circ}$ in CFD calculations. Furthermore, the pressure distribution is steeper in the calculation close to $\theta_{\min }$, and flatter at the upper side and the lower side. 


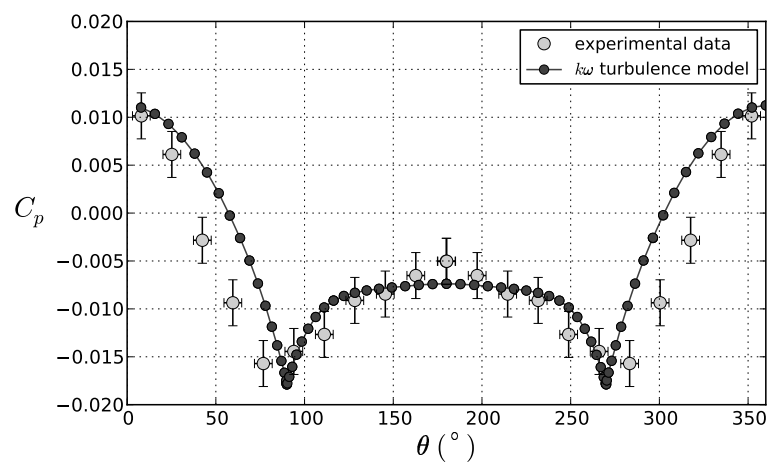

Fig. 11. Mean pressure distribution around the cylinder at a yaw angle $\alpha=5^{\circ}$.

\section{DISCUSSION}

\section{Velocity flow.}

As pointed out in Fig. 7, even though the main trends of the flow field are predicted by the present RANS simulations, significant discrepancies are still observed in the vicinity of the cylinder wall. The velocity deficit area is larger in RANS calculations compared to those observed in the experiments: it turns out that the boundary layer thickness provided by the simulations is overestimated. For such flow configuration, spatial flow development in the streamwise direction is driven by the boundary layer : a laminar turbulent transition occurs near the upstream tip of the cylinder and is followed by the emergence of a spatially developing turbulent boundary layer further downstream.

Unfortunately, such a flow patter falls outside the scope of calculations based on RANS turbulence modelling, and specific mechanisms such as the transition to turbulence in a boundary layer are clearly out reach. Closures of Reynoldsaveraged equations are indeed designed and calibrated for fully turbulent steady flows. As a consequence, during a RANS simulation, all boundary layers are assumed to be fully turbulent. The dissipation is overestimated, and so is the cylinder wake thickness.

\section{Pressure.}

The plot of the lift coefficient as a function of the yaw angle in Fig. 5 display a remarkable feature: despite the inability of RANS calculations to reproduce the turbulent boundary layer details, a fair collapse between simulations and experiments is observed for small enough angles $\left(\alpha<5^{\circ}\right)$. One could state that for low angles of attack, the lift coefficient mainly depends on the main flow features, so that the details close to the solid walls would not have a significant influence. Further investigations are nonetheless required to further assess this assumption. 
According to the experiments, for larger yaw angles $\left(\alpha>5^{\circ}\right)$ flow separation is expected with a loss of linearity between the lift coefficient and the angle $\alpha$. The RANS simulations seem to miss the onset of this new flow regime since the lift force remains a linear function of the angle of inclination over the range of angles investigated $\left(0^{\circ}<\alpha<15^{\circ}\right)$.

The CFD data are however obtained thanks to a steady RANS model which is not ideal for predicting unsteady turbulent phenomena such as the flow separation in the wake of a cylinder. In addition, the flow structure is likely to be threedimensional while the $k-\omega$ turbulence model is isotropic. Though they have not been assessed here, unsteady RANS, second-order closures (i.e. anisotropic) might yield more consistent results.

\section{CONCLUSION}

Experiments and CFD calculations have been carried out to determine the lift force exerted on a cylinder in nearaxial flow. The experimental results are consistent with the results obtained by Ersdal \& Faltinsen [6] and with previous results [12], namely, for inclination angles lower than $5^{\circ}$, the lift force appears proportional to the inclination angle. The velocity pattern and the pressure distribution around the cylinder indicate that the lift force is somehow related to a gradual velocity deficit at the lower side, without any clear flow separation.

The CFD computations and the experiments exhibit a reasonable agreement with respect to the minimum and the maximum pressure. The measured velocity deficit is predicted by the computation but its thickness is overestimated. This can be explained by the fact that the RANS modeling is designed for fully turbulent flow, with an isotropic turbulence model.

From a global point of view, the lift forces obtained by RANS CFD calculations are close to the experimental results for inclinations lower than $5^{\circ}$. Despite some modeling inaccuracies close to the wall, the RANS calculations seem able to predict the general trend of the lift force for small angles of inclinations.

Further work is needed to determine the range of validity of the quasi-static approach. The damping forces of a cylinder oscillating in axial flow should be investigated for low oscillation frequencies $(f<U / L)$ and low lateral velocities or low instantaneous angle $\left(\alpha(t)<5^{\circ}\right)$ and then compared to the normal force values predicted with the quasi-static approach.

\section{References}

[1] J.R. Morison, M. O. J. J., and Schaaf, S., 1950. "The force exerted by surface waves on piles”. Petroleum Transactions, AIME, 189, pp. 149-154.

[2] Sarpkaya, T., 2001. "On the force decompositions of Lighthill and Morison”. Journal of Fluids and Structures, 15, pp. $227-233$. 
[3] Sarpkaya, T., 2004. "A critical review of the intrinsic nature of vortex-induced vibrations". Journal of Fluids and Structures, 19(4), pp. 389-447.

[4] Sarpkaya, T., 1986. "Force on a circular cylinder in viscous oscillatory flow at low keulegancarpenter numbers". Journal of Fluid Mechanics, 165, pp. 61-71.

[5] Taylor, G., 1952. "Analysis of the swimming of long and narrow animals". Proceedings Royal Society of London, 214, pp. $158-183$.

[6] Ersdal, S., and Faltinsen, O., 2006. "Normal forces on cylinders in near-axial flow". Journal of Fluids and Structures, 22(8), pp. 1057-1077.

[7] Blevins, R., 1990. Flow-induced vibration. Van Nostrand Reinhold.

[8] Païdoussis, M., 2004. Fluid-structure interactions: slender structures and axial flow. Fluid-structure interactions. Academic Press.

[9] Archambeau, F., Sakiz, M., and Namane, M., 2004. "Code saturne: a finite volume code for turbulent flows”. Int. J. Finite Vol., 1(1), pp. 1-62.

[10] Zdravkovich, M., 1997. Flow around circular cylinders, oxford university press, new york ed., Vol. 1.

[11] Païdoussis, M. P., Price, S. J., and de Langre, E., 2011. Fluid-Structure Interactions: Cross-Flow-Induced Instabilities, cambridge university press, cambridge ed.

[12] Divaret, L., Cadot, O., Doaré, O., Moussou, P., and Berland, J., 2012. "Normal forces exerted upon a long cylinder oscillating in an axial flow". Proceedings of the 10th International conference on Flow-induced vibration. 